\title{
Diseño y aplicación de pilotes CFA para una central eléctrica en Coronel, Chile
}

\section{Design and implementation of CFA piles for a power plant in Coronel, Chile}

Fecha de entrega: 30 de octubre 2013 Fecha de aceptación: 21 de marzo 2014

\section{Dennis Raddatz ${ }^{1}$, Rodrigo Miranda ${ }^{2}$ y Oscar Taiba ${ }^{1}$}

${ }^{1}$ Ferrara - Proyectos Especiales, Rengo 1270, Nuñoa, Chile, draddatzalta@engin.umass.edu, oscartaiba@ferrara.cl

${ }^{2}$ Mageba USA, 575 Lexington Avenue, New York, NY 10022, USA, rmiranda@magebausa.com

El objetivo de este estudio es describir el sistema de pilotes CFA (Continuous Flight Auger) y mostrar un caso de aplicación en un proyecto de central eléctrica en Coronel, Chile. La ventaja principal de este método es la rapidez de ejecución de los pilotes, lo que se traduce en mayores rendimientos y menores costos. En Chile este método aún no se ha masificado, a pesar de que esta tecnología es popular en otros países y que condiciones de suelo favorables para su ejecución se encuentran con frecuencia a lo largo del país. En este artículo se presentan las fortalezas y debilidades del método, las condiciones geotécnicas favorables y desfavorables para su desarrollo, y el equipamiento, materiales y procedimientos necesarios para su ejecución. Además se describe el procedimiento para estimar la capacidad axial de los pilotes en los suelos donde es más común su ejecución. Finalmente, se presenta un caso aplicado en la región del Bío Bío, siguiendo los métodos y recomendaciones de normativas internacionales descritas previamente.

Palabras clave: pilote CFA, hélice continua, resistencia de pilote, pilote de fuste
The purpose of this study is to present the CFA piles system (Continuous Flight Auger) and to show a case of application for a power station project in Coronel, Chile. The main advantage of this method is the fast execution of the piles, which results in higher yields and lower costs. Even though this technology is popular in other countries and there are favourable soil conditions for its implementation in Chile, this method is not yet commonly used in the country. This paper describes the method's strengths and weaknesses, the favourable and unfavourable geotechnical conditions for its development, and the equipment, materials, and procedures necessary for its implementation. The paper also shows the procedure for estimating the axial capacity of piles in soils where the execution of the method is more common. Finally, an applied case in the Bio Bio Region is presented, following the methods of international regulations and recommendations previously described for the estimation of axial capacity on piles.

Keywords: CFA pile, continuous flight auger, pile capacity, friction pile

\section{Introducción}

La interacción entre la tecnología y el diseño ha jugado un papel preponderante en la evolución de los sistemas de pilotaje. Es precisamente esta interacción la que permite que la popularidad del sistema CFA (Continuous Flight Auger) comenzara a aumentar a fines de la década de los ochenta.
En la ejecución de pilotes CFA, la perforación es realizada a través de una hélice continua, sin necesidad de usar protección de las paredes durante la excavación. Además, el hormigonado se realiza mientras se produce el retiro de la hélice, lo que se traduce en un sistema más rápido que en los sistemas convencionales de ejecución de pilotes. 
El sistema CFA basa su creciente aceptación en el mercado comercial en su alta tasa de producción y su economía, es por esto que su uso se ha popularizado en EEUU y países europeos. En Chile este método aún no se ha masificado, a pesar de que los pilotes CFA se pueden usar para fundaciones o pantallas de retención de suelo. Además los suelos donde su ejecución es factible se encuentran con facilidad en diferentes partes del país.

En este trabajo se busca entregar información respecto al sistema de pilotes CFA, sus ventajas y desventajas, así como también las condiciones favorables y desfavorables para su construcción, siendo un factor preponderante en este ítem la tipología del suelo. Además, se presentarán los requerimientos y procedimientos para implementar dicho sistema en obra.

Se presenta también una revisión de la normativa internacional vigente para la estimación de la capacidad axial de los pilotes CFA, específicamente las normas FHWA 1999 (O’Neill y Reese, 1999) y DIN 4014 (DIN, 1990). Se acota el análisis a los suelos típicos donde se utiliza este tipo de pilotes. Se estudia un caso práctico en la región del Bío Bío, donde se determina la capacidad axial de un pilote CFA por medio de las normas descritas previamente. Finalmente, se entregan las conclusiones sobre las condiciones para el uso de pilotes CFA en Chile, y sobre los resultados obtenidos del uso de las normativas para el cálculo de capacidad axial.

\section{Descripción de pilotes CFA}

El sistema de pilotes CFA es un tipo de fundación profunda donde la perforación es realizada a través de una hélice continua hasta la profundidad final (Brown et al., 2007). En este proceso no es necesario contar con protección para mantener la estabilidad de la perforación, ya que los álabes de las hélices se llenan de material y proporcionan el soporte lateral necesario (Figura 1a). Al mismo tiempo que la hélice es retirada de la perforación, ésta se llena con hormigón que pasa a través de la hélice hueca hasta la base de la perforación. El soporte de la perforación se da de forma continua, tanto por el hormigón como por la hélice (Figura 1b). Inmediatamente después de que es retirada la hélice y con la perforación llena de hormigón, se introduce la armadura de acero (Figura 1c).

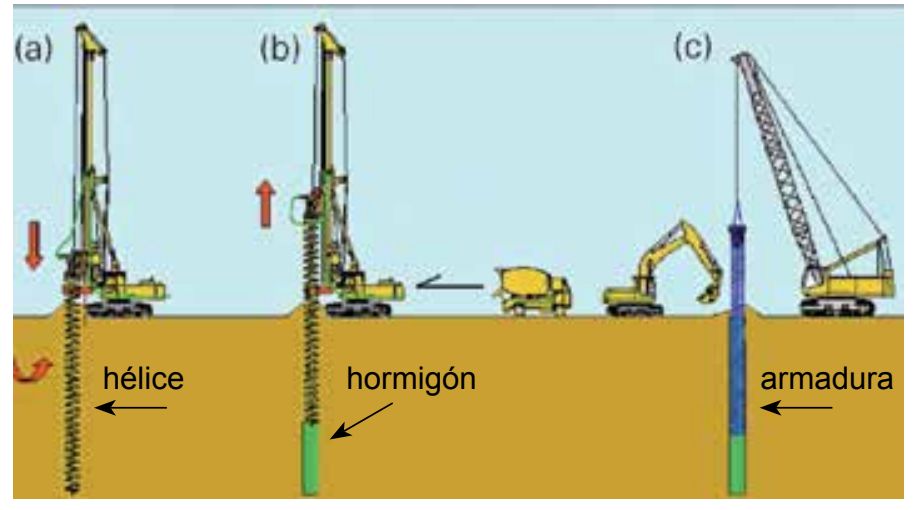

Figura 1: Esquema ejecución pilotes CFA (Brown et al., 2007)

El método usado por el sistema CFA ha sido posible de ejecutar por los avances en las técnicas de perforación, especialmente por la capacidad de torque y empuje descendente generados por los equipos de perforación (Coduto et al., 2011). A finales de los años 80 y principios de los 90, los equipos de perforación pesaban entre $30 \mathrm{y}$ $40 \mathrm{~T}$, con alturas de mástil de 10 a $15 \mathrm{~m}$ y con capacidades de torque de 10 a $15 \mathrm{Tm}$. En el mercado actual es posible encontrar una gran variedad de equipos de perforación, como la BG50 de Bauer, con torque de $46.8 \mathrm{Tm}$, altura de mástil de $30 \mathrm{~m}$ y peso de $250 \mathrm{~T}$ (Bauer, 2011).

Usualmente el rango de diámetros de pilotes CFA es de 40 a $90 \mathrm{~cm}$, mientras que el rango de profundidad es de los 8 a $30 \mathrm{~m}$ (Candogan, 2009). Producto del bajo diámetro, estos pilotes funcionarán de forma aislada para cargas bajas, siendo necesario usarlos en grupo para cargas mayores. Los pilotes CFA se pueden utilizar perfectamente para muros de pantallas de pilotes, ya sea discontinuos, continuos o secantes (Stocker y Waltz, 2003).

Las principales ventajas de este método son su rapidez y menor costo al compararse con otras alternativas convencionales (Pilotes Terratest, 2009). El tiempo de ejecución puede verse reducido a menos de la mitad en algunos casos. El rendimiento promedio de una máquina con este método varía entre 100 a $250 \mathrm{ml} /$ día. Los pilotes CFA presentan una gran ventaja en suelos inestables y bajo napa respecto a los pilotes excavados de forma convencional, evitándose los problemas del hormigonado bajo agua (Tomlinson y Woodward, 2007). El perforado se realiza de forma silenciosa con bajas vibraciones, ideal para zonas urbanas. 
La principal desventaja del método es que requiere mayor energía para el torque y empuje descendente que otras técnicas (Brown et al., 2007). Además, su uso está limitado hasta rocas meteorizadas. Por otro lado, es más complejo definir la estratificación real del terreno, ya que la profundidad del material extraído no puede ser comprobada (Terzaghi et al., 1996).

Los suelos en que se obtienen buenos resultados ejecutando pilotes CFA corresponden a arcillas de mediana a gran rigidez, arenas cementadas, suelos residuales, arenas limosas y en general, suelos que no se presenten extremadamente duros (Pilotes Terratest, 2009). Se deben evitar suelos excesivamente blandos, ya que se produce sobreconsumo de hormigón producto de la inestabilidad de la excavación (Brown et al., 2007). En arenas sueltas o muy limpias bajo napa, se puede producir una extracción excesiva de suelo. Lo mismo puede ocurrir cuando el estrato de arena está bajo un estrato de arcilla rígida. El avance lento de penetración en arcilla puede causar aflojamiento de un estrato de arena cuando éste es alcanzado, lo cual puede resultar en una excesiva extracción de arena y disminución de soporte.

En los pilotes CFA no se tiene una alta resistencia por punta, esto se produce porque su ejecución en estratos duros no es posible y los diámetros que se pueden ejecutar con este método son más restringidos que con otras técnicas. El mayor aporte para la resistencia axial lo entrega el fuste, esto se podrá apreciar en detalle en el caso aplicado, donde se calcula la resistencia axial de un pilote CFA.

\section{Equipamiento de construcción}

Existen distintas alternativas de maquinarias para la construcción de pilotes perforados. Su elección depende de la disponibilidad y el tipo de trabajo que se ejecute (Brown et al., 2007).

- Equipo de perforación montado en grúa oruga. La hélice continua es introducida en el suelo accionada por un motor hidráulico instalado en la parte superior de la hélice. La guía del pilote provee verticalidad a la hélice, además sostiene el motor hidráulico que ejerce el torque a la hélice. El brazo de torque o estabilización sostiene la guía del pilote para absorber el torque producido al realizar la perforación. El destorcedor en la punta de la hélice permite que ésta gire libremente sin desconectar la línea de hormigón.

- Equipo de perforación de altura restringida. Este equipo se monta normalmente en camiones u orugas y tiene una gran capacidad de movimiento. Se utilizan secciones de hélices para lograr la profundidad necesaria.

- Equipo de perforación montado en orugas (piloteras), como se muestran en la Figura 2a. Esta maquinaria es la más utilizada para la construcción de pilotes CFA en Europa y EEUU. Básicamente es la misma máquina que realiza pozos perforados en el sistema tradicional, pero en vez de un Kelly largo o brazo de torque extensible, posee uno más pequeño o simplemente no lo posee, dando lugar a la hélice continua.

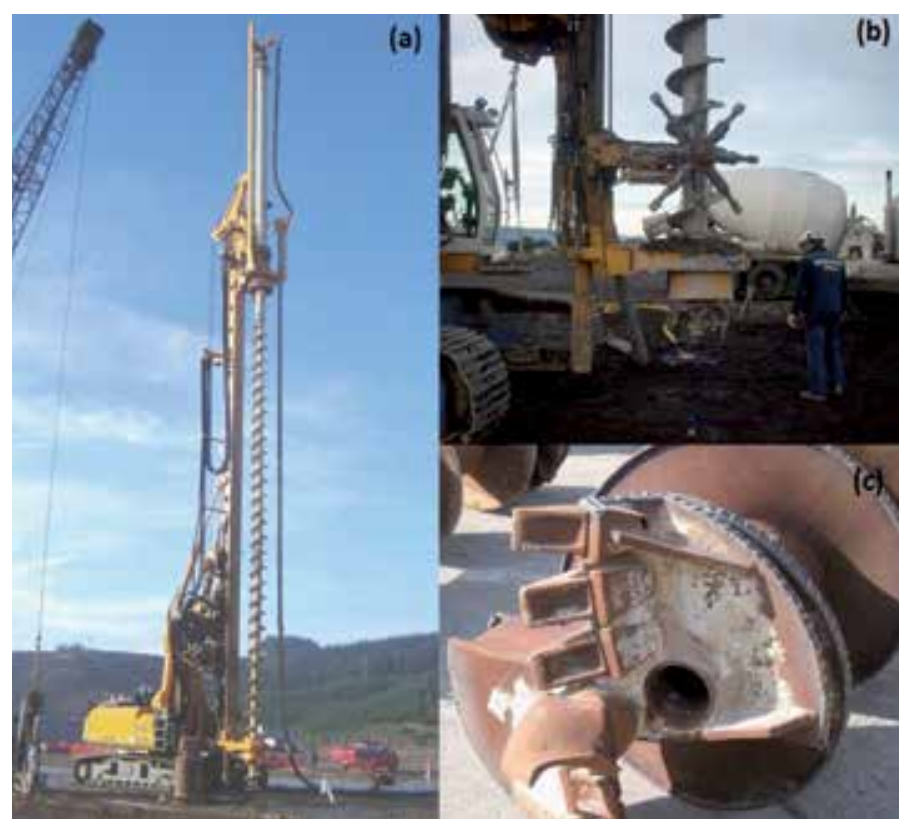

Figura 2: a) Ejecución pilote CFA de $18 \mathrm{~m}$ (Pilotes Terratest, 2009), b) limpieza de hélice (Pilotes Terratest, 2009) y c) punto de descarga de hormigón en el fondo (Brown et al., 2007)

La selección del tamaño de los álabes de una hélice es importante, ya que para un determinado tipo de suelo, un excesivo ancho en los álabes puede provocar una sobre excavación del pozo. Un accesorio de vital importancia a la hora de retirar la hélice del pozo es el limpiador de hélice (Figura 2b), el cual remueve el material extraído del pozo.

Para la mayoría de las construcciones de pilotes CFA, el 
hormigón se bombea a presión desde la parte superior de la hélice y a través de ella. El punto de descarga del hormigón se encuentra en la parte inferior de la hélice (Figura 2c). El equipo de bombeo debe desarrollar presiones de bombeo sobre los 2.4 MPa. La presión de bombeo debe superar la presión hidrostática del hormigón, además se debe ajustar la velocidad de retiro de la hélice a la velocidad de suministro de hormigón, de forma de evitar la formación de vacíos o disminución de sección en el pilote (Kempfert et al., 2003).

\section{Hormigón y armadura}

Los requerimientos típicos para un hormigón utilizado para la construcción de pilotes CFA corresponden a un esfuerzo de compresión de 20 a $30 \mathrm{MPa}$; un rango para el asentamiento de cono del hormigón de $20 \pm 2.5 \mathrm{~cm}$; un rango para la temperatura de colocación del hormigón entre 4 y $38^{\circ} \mathrm{C}$; y un tamaño máximo para el agregado grueso típicamente limitado a $10 \mathrm{~mm}$. Se recomienda que el agregado sea redondeado lo que favorece la trabajabilidad de la mezcla y el bombeo (Brown et al., 2007).

El acero de refuerzo para las armaduras de pilotes CFA debe cumplir con la norma NCh204 (INN, 2006). Las barras no pueden ser soldadas para construir las armaduras, ya que se reduce su capacidad de fluencia. En lugares donde existe peligro de corrosión es posible usar acero galvanizado o con cubiertas epóxicas.

\section{Capacidad axial estática de pilotes CFA}

En esta sección se describen los métodos para estimar la capacidad axial de los pilotes CFA según la norma estadounidense FHWA 1999 (O’Neill y Reese, 1999) y la norma alemana DIN 4014 (DIN, 1990) para los suelos donde más comúnmente se ejecutan los pilotes CFA, es decir, suelos cohesivos y granulares, evitándose estratos muy firmes o rocas. Estas normativas internacionales se usan en Chile, ya que en el país no existe una norma específica para la ejecución de pilotes.

\section{FHWA 1999}

Según la FHWA 1999 (O’Neill y Reese, 1999), la resistencia nominal total a compresión axial $R_{T N}$ de un pilote CFA se calcula como la combinación de la resistencia nominal por fuste $R_{S N}$ y por punta $R_{B N}$ :

$$
R_{T N}=R_{S N}+R_{B N}=\pi D \sum_{i=1}^{n} z_{i} f_{\max i}+\pi \frac{D^{2}}{4} q_{\max }
$$

donde $z_{i}$ es la longitud del estrato $i, \mathrm{y} f_{\max i}$ es la resistencia última por fuste del estrato $i, q_{\max }$ es la resistencia última de la base del pilote y $D$ es el diámetro del pilote en ese punto (Figura 3).

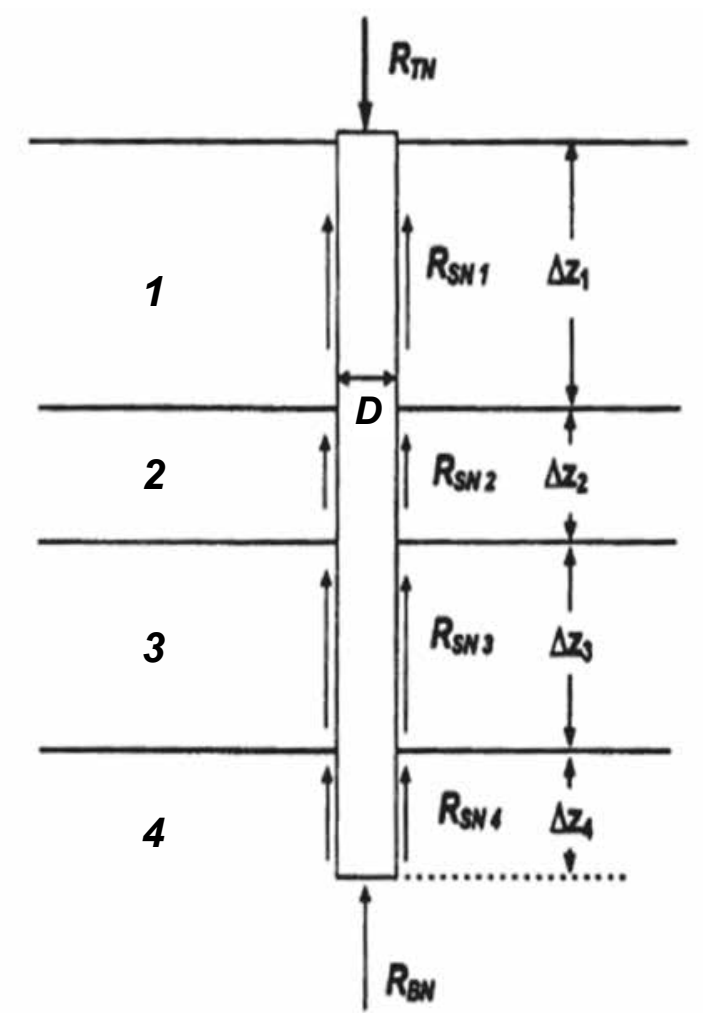

Figura 3: Distribución de resistencias de un pilote (O’Neill y Reese, 1999)

La componente de la resistencia por fuste se moviliza relativamente con desplazamientos verticales del pilote muy pequeños, normalmente inferior a $1 \mathrm{~cm}$. La componente de la resistencia por punta está plenamente movilizada con grandes desplazamientos, por lo general en el rango de 5 a $10 \%$ del diámetro del pilote. La resistencia de un pilote CFA a cualquier desplazamiento vertical deseado puede ser encontrada usando la relación de carga y asentamiento de la Figura 4. El procedimiento utilizado para definir la resistencia última de un pilote perforado en compresión se define a partir de los siguientes criterios de falla:

- Carga de punzonamiento para suelos cohesivos. 
- Carga correspondiente a un asentamiento del pilote de un $5 \%$ del diámetro de la base para suelos granulares.
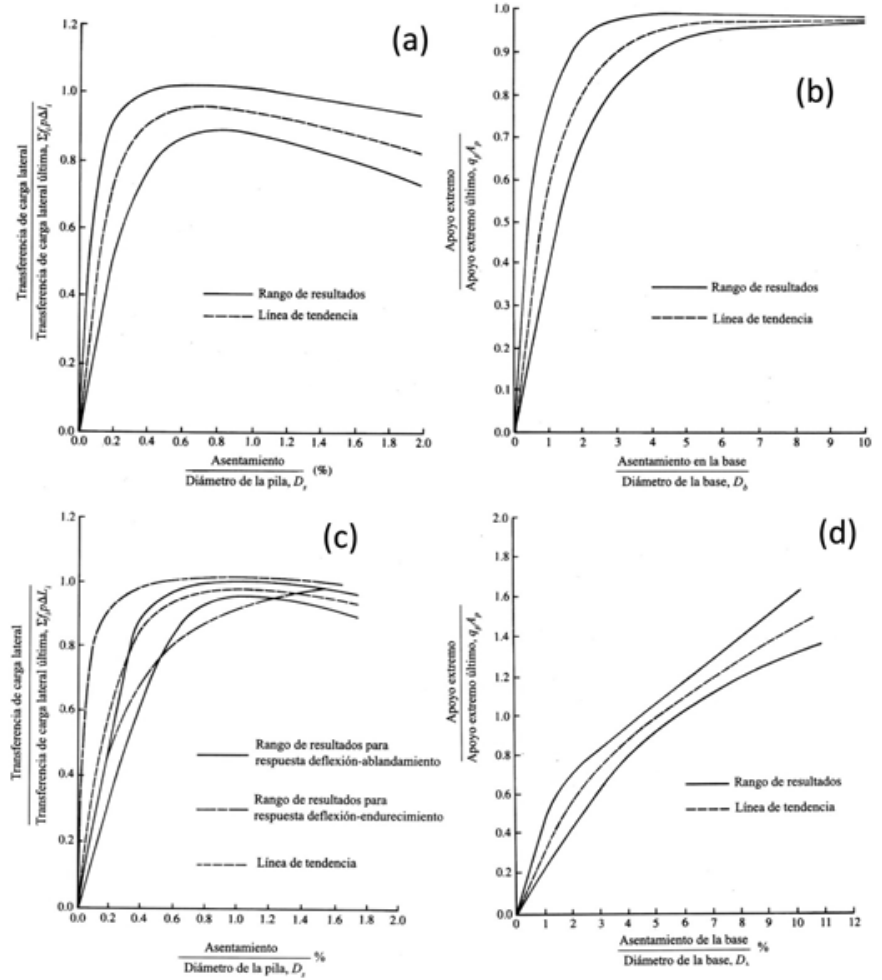

Figura 4: Carga normalizada por carga última versus asentamiento normalizado por diámetro de la base, a) carga lateral para suelo cohesivo, b) carga en la base para suelo cohesivo, c) carga lateral para suelo sin cohesión y d) carga en la base para suelo sin cohesión (Reese y O’Neill, 1989).

El procedimiento de diseño para pilotes CFA está organizado según el tipo de suelo. La Tabla 1 muestra la clasificación de suelos presentada por FHWA 1999.

Tabla 1: Clasificación de suelos según FHWA 1999 (Reese y O’Neill, 1999)

\begin{tabular}{|l|c|c|}
\hline Tipo de suelo & $s_{u}, \mathrm{kPa}$ & $\mathrm{N}_{\mathrm{SPT}}$, golpes $/ 0.3 \mathrm{~m}$ \\
\hline $\begin{array}{l}\text { Cohesivo: arcilla o } \\
\text { limo plástico }\end{array}$ & $\leq 250$ & - \\
\hline $\begin{array}{l}\text { No cohesivo o granular: } \\
\text { arena, grava o limo no } \\
\text { plástico }\end{array}$ & - & $\leq 50$ \\
\hline
\end{tabular}

\section{Suelos cohesivos}

En suelos cohesivos la resistencia última por fuste $f_{\max i}$ para un determinado segmento de pilote se calcula como:

$$
f_{\max i}=\alpha s_{u}
$$

donde $s_{u}$ es la resistencia al corte no drenada del suelo y $\alpha$ el factor de reducción del esfuerzo de corte que se define como:

$$
\begin{array}{ll}
\alpha=0.55 & \text { para } s_{u} / p_{a} \leq 1.5 \\
\alpha=0.55-0.1\left(s_{u} / p_{a}-1.5\right) & \text { para } 1.5 \leq s_{u} / p_{a} \leq 2.5
\end{array}
$$

donde $p_{a}$ es la presión atmosférica igual a $100 \mathrm{kPa}$. Si la parte inferior de un pilote es soportada por un suelo cohesivo, la contribución del fuste a la capacidad de fondo del pilote es nula para una altura igual a un diámetro desde la base del pilote. Si el suelo es cohesivo en la superficie, no se considera el aporte de la resistencia por fuste en la cabeza del pilote por la mayor distancia entre $1.5 \mathrm{~m}$ medidos desde la superficie del suelo y la profundidad de la zona de cambios de humedad estacionales. La resistencia última de la base $q_{\max }$ se calcula como:

$$
q_{\max }=s_{u} N_{c}^{*}
$$

donde $s_{u}$ es la resistencia al corte no drenada en la zona de la punta y $N_{c}{ }^{*}$ es el factor de capacidad de soporte que se define como:

$$
\begin{aligned}
& N_{c}^{*}=9 \quad \text { para } 200 \mathrm{kPa} \leq s_{u} \leq 250 \mathrm{kPa} \mathrm{y} L \geq 3 D \\
& N_{c}^{*}=4 / 3\left[\ln \left(I_{r}+1\right)\right] \quad \text { para } s_{u} \leq 200 \mathrm{kPa} \text { y } L \geq 3 D
\end{aligned}
$$

donde $L$ es el largo del pilote e $I_{r}$ es el índice de rigidez del suelo.

\section{Suelos no cohesivos}

La resistencia última por fuste $f_{\max }$ para un determinado segmento de pilote " $i$ ", se calcula como:

$$
f_{\max i}=\beta_{i} \sigma_{v i}^{\prime} \leq 200 \mathrm{kPa}
$$

donde $\sigma_{v i}^{\prime}$ es la tensión vertical efectiva en el centro del estrato $i, \beta_{i}$ es el factor de correlación adimensional entre la tensión vertical efectiva $\sigma_{v i}^{,} \mathrm{y} f_{\max }$ para el estrato $i$, con un valor máximo de 1.2 en arenas y 1.8 en arenas con gravas o gravas y con un valor mínimo de 0.25 . Usando el número de golpes $/ 0.3 \mathrm{~m}$ del ensayo SPT corregido a un $60 \%$ de la energía $\mathrm{N}_{60}$, se tiene:

Para arenas:

$$
\begin{array}{ll}
\beta_{i}=1.5-0.245 \sqrt{z_{i}} & \text { para } 15 \leq \mathrm{N}_{60} \leq 50 \text { golpes } / 0.3 \mathrm{~m} \\
\beta_{i}=\left(\frac{N_{60}}{15}\right)\left(1.5-0.245 \sqrt{z_{i}}\right) & \text { para } \mathrm{N}_{60}<15 \text { golpes } / 0.3 \mathrm{~m}
\end{array}
$$


Para arenas con gravas o gravas:

$\beta_{i}=2.0-0.15 z_{i}^{0.75} \quad$ para $15 \leq \mathrm{N}_{60} \leq 50$ golpes $/ 0.3 \mathrm{~m}$

donde $\mathrm{N}_{60}$ es el valor de diseño para la cantidad de golpes del SPT con un $60 \%$ de eficiencia del martillo, no corregido por profundidad, saturación o finos, representativo del estrato o segmento $i$ y $z_{i}$ es la distancia vertical desde la superficie del terreno al centro de la capa $i$ en metros. La resistencia última de la base $q_{\max }$ en $\mathrm{kPa}$ en suelos no cohesivos, caracterizados por un SPT $\mathrm{N}_{60}$ que no supera los 50 golpes $/ 0.3 \mathrm{~m}$, se calcula como:

$$
q_{\max }=57.5 \mathrm{~N}_{60} \leq 2900 \mathrm{kPa}
$$

\section{DIN 4014}

La capacidad a compresión de pilotes perforados descrita en la norma DIN 4014 (DIN, 1990), se calcula de acuerdo a una curva empírica de carga-asentamiento sobre la base de pruebas de carga de pilotes. La resistencia de un suelo no cohesivo se puede expresar con suficiente precisión en términos de la resistencia de punta $q_{c}$ del ensayo CPT y en el caso de suelo cohesivo, en términos de la resistencia al corte no drenada $s_{u}$.

\section{Resistencia de punta de un pilote}

Las especificaciones para la resistencia de punta se señalan en las Tablas 2 y 3 para distintos niveles de asentamiento $s$ del pilote de diámetro $D$, donde se asume que el pilote falla para $s=0.1 D$. Valores intermedios pueden ser obtenidos por interpolación lineal.

Tabla 2: Resistencia de punta $\sigma_{\mathrm{s}}$ en MPa en suelo no cohesivo (DIN, 1990)

\begin{tabular}{|c|c|c|c|c|}
\hline \multirow{2}{*}{$s / D$} & \multicolumn{4}{|c|}{ Resistencia promedio $q_{c}, \mathrm{MPa}$} \\
\cline { 2 - 5 } & 10 & 15 & 20 & 25 \\
\hline 0.02 & 0.7 & 1.05 & 1.4 & 1.75 \\
\hline 0.03 & 0.9 & 1.35 & 1.8 & 2.25 \\
\hline 0.1 & 2.0 & 3.0 & 3.5 & 4.0 \\
\hline
\end{tabular}

Tabla 3: Resistencia de punta $\sigma_{\mathrm{s}}$ en MPa en suelo cohesivo (DIN, 1990)

\begin{tabular}{|c|c|c|}
\hline \multirow{2}{*}{$s / D$} & \multicolumn{2}{|c|}{ Resistencia al corte no drenada $s_{u^{,}} \mathrm{kPa}$} \\
\cline { 2 - 3 } & 100 & 200 \\
\hline 0.02 & 0.35 & 0.9 \\
\hline 0.03 & 0.45 & 1.1 \\
\hline 0.1 & 0.8 & 1.5 \\
\hline
\end{tabular}

La Tabla 4 muestra una relación aproximada entre SPT y $q_{c}$ para usar en caso de no contar con valores de este último. El método está calibrado para el CPT y esta conversión debería ser verificada debido a la variabilidad de los resultados del ensayo SPT.

Tabla 4: Factores de conversión para ensayos SPT (DIN, 1990)

\begin{tabular}{|l|c|}
\hline Tipo de suelo & $q_{c} / N_{30}{ }^{*}, \mathrm{MPa}$ \\
\hline $\begin{array}{l}\text { Fino a medio o arena levemente } \\
\text { limosa }\end{array}$ & $0.3 \mathrm{a} 0.4$ \\
\hline Arena o arena con grava leve & 0.5 a 0.6 \\
\hline $\begin{array}{l}\text { Arena de granulometría } \\
\text { discontinua }\end{array}$ & 0.5 a 1.0 \\
\hline Grava arenosa o grava & 0.8 a 1.0 \\
\hline
\end{tabular}

* Número de golpes por $30 \mathrm{~cm}$ de penetración

\section{Resistencia por fuste de un pilote}

La resistencia última por fricción del fuste de un pilote individual se debe asumir como las dadas en las Tabla 5 y 6 . Valores intermedios pueden ser obtenidos por interpolación lineal. El aumento de la resistencia por fuste es lineal hasta alcanzar el último valor de asentamiento calculado $s_{r g}$ (Figura 5).

Tabla 5: Resistencia última de fuste en suelo no cohesivo (DIN, 1990)

\begin{tabular}{|c|c|}
\hline $\begin{array}{c}\text { Resistencia promedio } q_{c}, \\
\text { MPa }\end{array}$ & $\begin{array}{c}\text { Resistencia última de } \\
\text { fuste } \tau_{m i}, \mathrm{MPa}\end{array}$ \\
\hline 0 & 0 \\
\hline 5 & 0.04 \\
\hline 10 & 0.08 \\
\hline$\geq 15$ & 0.12 \\
\hline
\end{tabular}


Tabla 6: Resistencia última de fuste en suelo cohesivo (DIN, 1990)

\begin{tabular}{|c|c|}
\hline $\begin{array}{c}\text { Resistencia al corte no } \\
\text { drenada } s_{u}, \mathrm{MPa}\end{array}$ & $\begin{array}{c}\text { Resistencia última de } \\
\text { fuste } \tau_{m i}, \mathrm{MPa}\end{array}$ \\
\hline 25 & 0.025 \\
\hline 100 & 0.04 \\
\hline$\geq 200$ & 0.06 \\
\hline
\end{tabular}

\section{Curva de carga-asentamiento}

Una curva similar a la que se muestra en la Figura 5, se establecerá a partir de los resultados obtenidos con las tablas anteriores (Tablas 2 a 6). La resistencia por punta es función del asentamiento $s_{g}$ que está dado por:

$$
s_{g}=0.1 \mathrm{D}
$$

La resistencia última por fricción del fuste es función del asentamiento $S_{r g}$ que está dado por:

$$
s_{r g}=0.5 Q_{r g}+0.5 \leq 3 \mathrm{~cm}
$$

donde $Q_{r g}$ está en MN. La curva carga-asentamiento debe ser calculada usando la siguiente ecuación:

$$
Q(s)=Q_{s}(s)+Q_{r}(s)=A_{f} \sigma_{s}(s)+\sum_{i=1} A_{m i} \tau_{m i}(s)
$$

donde $Q_{s}(s)$ es la resistencia de punta en función del asentamiento de la cabeza del pilote (Tablas 2 y 3 ), $Q_{r}(s)$ es la resistencia del fuste en función del asentamiento de la cabeza del pilote (Tablas 5 y 6 ).

$$
Q_{r g}=\sum_{i=1} A_{m i} \tau_{m i}(s)
$$

$A_{f}$ es el área de la base del pilote, $\sigma_{s}(s)$ es la resistencia de punta en función del asentamiento de la cabeza del pilote (Tablas 2 y 3 ), $A_{m i}$ es el área de la superficie exterior del pilote en la longitud embebida en el estrato y $\tau_{m i}(s)$ es la resistencia por fricción del fuste en función del asentamiento de la cabeza del pilote (Tabla 5 y 6 ).

\section{Caso de aplicación práctica}

En esta sección se presenta un ejemplo que ilustra la estimación de la capacidad axial de un pilote usando las normas FHWA 1999 (O’Neill y Reese, 1999) y la DIN

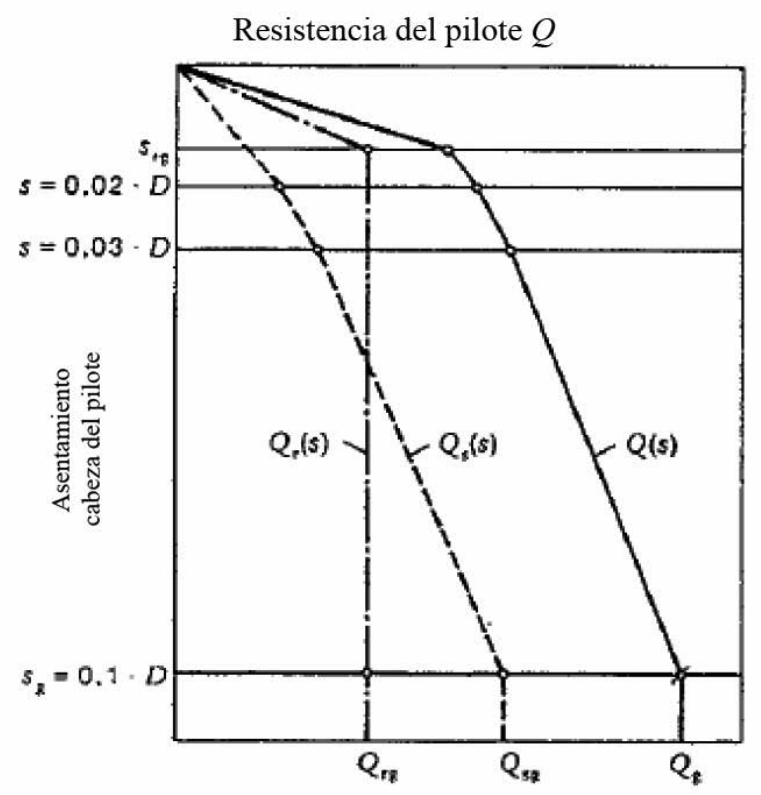

Figura 5: Curva de carga versus asentamiento en base a Tablas 2, 3,5 y 6 (DIN, 1990)

4014 (DIN, 1990) descritas en la sección anterior. Los datos utilizados corresponden a pilotes CFA realizados por Pilotes Terratest el año 2008. En total se construyeron 800 pilotes para una planta termoeléctrica a carbón de la empresa Colbún S.A en Coronel. Posteriormente, a las resistencias últimas calculadas se deben aplicar los factores de reducción de resistencia especificados según el tipo de diseño empleado. De manera de verificar la resistencia con las cargas de diseño.

\section{Datos del pilote y perfil del terreno}

Se considera la construcción de pilotes de $0.6 \mathrm{~m}$ de diámetro y $16 \mathrm{~m}$ de longitud. La Figura 6 muestra el perfil estratigráfico del terreno, el cual en los primeros metros presenta un material arcillo-arenoso, de consistencia blanda, pasando por un estrato limoso de consistencia dura para finalmente llegar a una arenisca de compacidad media que a mayor profundidad aumenta su cementación. Los datos del sondaje se muestran en la Tabla 7. El agua subterránea está a una profundidad de $2.8 \mathrm{~m}$ desde la superficie.

\section{Solución mediante FHWA 1999}

La Tabla 8 presenta los valores de los parámetros del suelo obtenidos a partir de ensayos SPT y de laboratorio. 


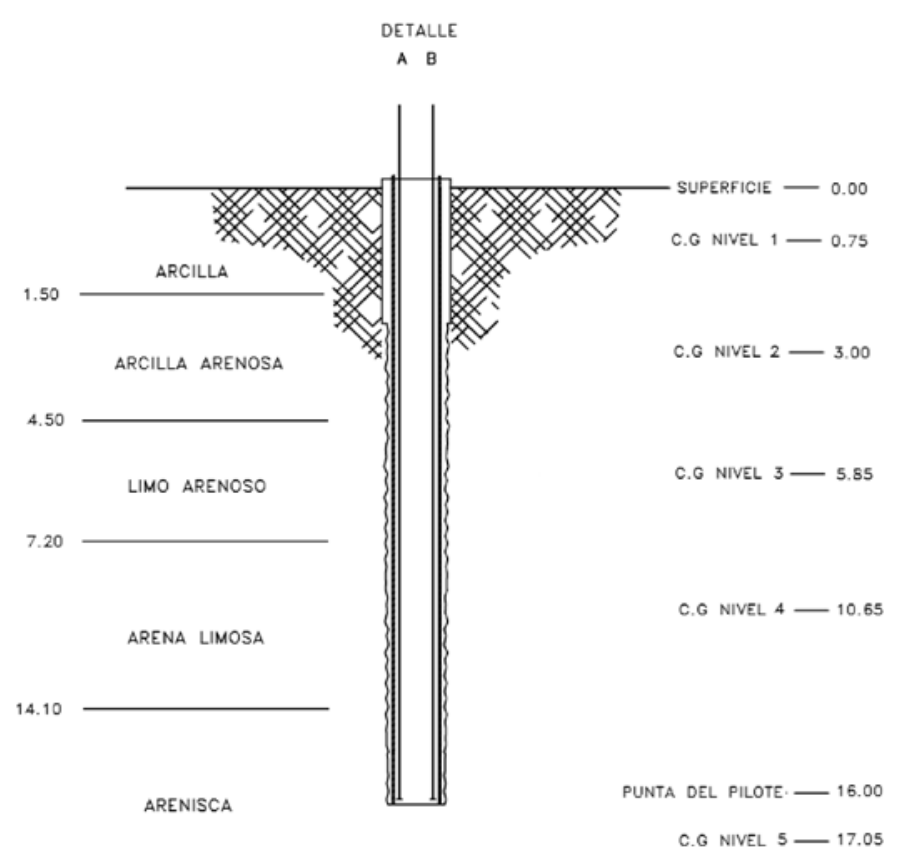

Figura 6: Perfil estratigráfico del terreno del proyecto en estudio

Tabla 7: Resultado sondaje SI-6 Proyecto Colbún (fuente: Pilotes Terratest)

\begin{tabular}{|c|c|c|c|c|c|c|c|}
\hline \multirow[t]{2}{*}{ Estrato } & \multirow[t]{2}{*}{$\begin{array}{l}\mathrm{de} \\
\mathrm{m}\end{array}$} & \multirow[t]{2}{*}{$\begin{array}{l}\mathrm{a} \\
\mathrm{m}\end{array}$} & \multirow[t]{2}{*}{$\begin{array}{l}\text { descripción visual del } \\
\text { terreno }\end{array}$} & \multicolumn{4}{|c|}{$\begin{array}{l}\text { Ensayo de } \\
\text { Penetración } \\
\text { Estándar }\end{array}$} \\
\hline & & & & N1 & N2 & $N 3$ & $N$ \\
\hline 1 & 0 & 1.5 & $\begin{array}{l}\text { arcilla arenosa, } \\
\text { consistencia blanda, } \\
\text { plasticidad baja y } \\
\text { humedad alta }\end{array}$ & 2 & 1 & 2 & 3 \\
\hline 2 & 1.5 & 4.5 & $\begin{array}{l}\text { arcilla arenosa, } \\
\text { plasticidad alta, } \\
\text { consistencia blanda y } \\
\text { humedad alta }\end{array}$ & 3 & 3 & 4 & 7 \\
\hline 3 & 4.5 & 7.2 & $\begin{array}{l}\text { limo algo arenoso } \\
\text { de consistencia dura, } \\
\text { humedad alta y } \\
\text { plasticidad nula }\end{array}$ & 5 & 10 & 13 & 23 \\
\hline 4 & 7.2 & 14.1 & $\begin{array}{l}\text { arena muy fina, limosa, } \\
\text { compacidad media, } \\
\text { humedad alta y nula } \\
\text { plasticidad }\end{array}$ & 7 & 12 & 20 & 32 \\
\hline 5 & 14.1 & 20 & $\begin{array}{l}\text { arenisca arcillosa } \\
\text { levemente cementada }\end{array}$ & 18 & 16 & 16 & 32 \\
\hline
\end{tabular}

Tabla 8: Parámetros del suelo a partir de correlaciones con SPT

\begin{tabular}{|c|c|c|c|c|c|c|}
\hline Estrato & $N_{60}$ & $D R, \%$ & $\gamma_{d^{\prime}} \mathrm{kN} / \mathrm{m}^{3}$ & $w$ & $\gamma_{a p a^{\prime}} \mathrm{kN} / \mathrm{m}^{3}$ & $\sigma_{{ }_{v}}^{\prime} k P a$ \\
\hline 1 & 3 & 42 & 14.2 & 0.124 & 15.96 & 11.97 \\
\hline 2 & 7 & 48 & 14.8 & 0.136 & 16.81 & 48.48 \\
\hline 3 & 23 & 61 & 16.1 & 0.082 & 17.42 & 72.02 \\
\hline 4 & 32 & 67 & 16.4 & 0.094 & 17.94 & 114.15 \\
\hline 5 & 32 & 67 & 16.4 & 0.094 & 17.94 & 166.25 \\
\hline
\end{tabular}

Donde $\mathrm{N}_{60}$ es el número de golpes del SPT con un $60 \%$ de eficiencia del martillo, DR es la densidad relativa porcentual, $\gamma_{d}$ es el peso unitario seco del terreno, $w$ es la humedad del terreno, $\gamma_{\text {apa }}$ es el peso unitario aparente del terreno y $\sigma^{\prime}{ }_{\nu}$ es la tensión vertical efectiva del terreno.

Para el cálculo de la resistencia por fuste, se debe reconocer el tipo de suelo en el que se encuentra cada estrato. Los tres primeros estratos corresponden a un suelo cohesivo, por lo que se utilizan las ecuaciones (1) a (3). Los siguientes dos estratos corresponden a un suelo no cohesivo, por lo que se usan las expresiones (4) y (5).

Tabla 9: Resistencia por fuste en suelo cohesivo

\begin{tabular}{|c|c|c|c|c|c|c|}
\hline Estrato & $A_{m i^{\prime}}, \mathrm{m}^{2}$ & $S_{u^{\prime}} \mathrm{kPa}$ & $\alpha$ & $f_{\text {maxi }}, \mathrm{MPa}$ & $R_{S N^{\prime}} \mathrm{MN}$ & $R_{S N^{\prime}} \mathrm{T}$ \\
\hline 1 & 2.82 & 25 & 0.00 & 0.000 & 0.000 & 0.0 \\
\hline 2 & 5.65 & 40 & 0.55 & 0.022 & 0.124 & 12.4 \\
\hline 3 & 5.08 & 153 & 0.54 & 0.084 & 0.426 & 42.6 \\
\hline
\end{tabular}

Tabla 10: Resistencia por fuste en suelo no cohesivo

\begin{tabular}{|c|c|c|c|c|c|c|}
\hline Estrato & $A_{m i}, \mathrm{~m}^{2}$ & $\beta$ & $\sigma^{\prime}, \mathrm{MPa}$ & $f_{\text {maxi }}, \mathrm{MPa}$ & $R_{S N} \mathrm{MN}$ & $R_{S N} \mathrm{~T}$ \\
\hline 4 & 13.00 & 0.70 & 0.114 & 0.08 & 1.040 & 104 \\
\hline 5 & 11.12 & 0.49 & 0.166 & 0.08 & 0.903 & 90.3 \\
\hline
\end{tabular}

La resistencia por fuste total es de $R_{S N}=249.3 \mathrm{~T}$. Para el cálculo de la resistencia por punta se utiliza la expresion (5).

Tabla 11: Resistencia por punta en suelo no cohesivo

\begin{tabular}{|c|c|c|c|}
\hline$N_{60}$ & $q_{\text {max }}, \mathrm{MPa}$ & $R_{B N} \mathrm{MN}$ & $R_{B N} \mathrm{~T}$ \\
\hline 32 & 1.84 & 0.52 & 52.0 \\
\hline
\end{tabular}

La capacidad axial del pilote es de $R_{T N}=301.3 \mathrm{~T}$. Los desplazamientos verticales asociados a las resistencias 
últimas movilizadas por punta y fuste se obtienen de las curvas idealizadas mostradas en la Figura 4. Para obtener el desplazamiento movilizado por fuste para las resistencias calculadas anteriormente, se deben calcular las proporciones de resistencia de fuste en cada estrato respecto al total y luego, a partir de las curvas idealizadas, obtener el asentamiento.

Tabla 12: Asentamiento vertical del fuste según FHWA 1999

\begin{tabular}{|c|c|c|c|c|c|}
\hline $\begin{array}{l}\text { Tipo de } \\
\text { suelo }\end{array}$ & $R_{S N i}, \mathrm{MN}$ & $R_{S T} \mathrm{MN}$ & $R_{S N i} / R_{S T}$ & $s / D, \%$ & $s, \mathrm{~cm}$ \\
\hline Cohesivo & 0.550 & 2.493 & 0.221 & 0.141 & 0.085 \\
\hline $\begin{array}{l}\text { No } \\
\text { cohesivo }\end{array}$ & 1.943 & 2.493 & 0.779 & 0.779 & 0.467 \\
\hline & & & & Total & 0.552 \\
\hline
\end{tabular}

La FHWA 1999 considera que la capacidad última de resistencia en la base es movilizada por un asentamiento de la cabeza del pilote de un $5 \%$ del diámetro del pilote. Utilizando la curva mostrada en la Figura 4 para el asentamiento en un suelo no cohesivo movilizado por la punta del pilote, se obtienen los resultados mostrados en la Tabla 13.

Tabla 13: Asentamiento vertical en la base según FHWA 1999

\begin{tabular}{|l|c|c|c|c|c|}
\hline $\begin{array}{l}\text { Tipo de } \\
\text { suelo }\end{array}$ & $R_{B N} \mathrm{MN}$ & $R_{B T} \mathrm{MN}$ & $R_{B N i} / R_{B T}$ & $s / D, \%$ & $\begin{array}{c}s, \\
\mathrm{~cm}\end{array}$ \\
\hline $\begin{array}{l}\text { No } \\
\text { cohesivo }\end{array}$ & 0.52 & 0.52 & 1 & 5 & 3 \\
\hline
\end{tabular}

En la Figura 7 se muestra la curva de resistencia versus asentamiento del pilote basada en los cálculos realizados previamente.

\section{Solución mediante la norma DIN 4014}

Para suelos no cohesivos se utiliza la tabla que relaciona SPT y $q_{c}$, ya que no se contaba con resultados de ensayos CPT. En tanto para suelo cohesivo, se correlacionaron los valores de SPT con los valores de resistencia al corte no drenada $s_{u}$ correspondientes, ya que no se contaba con ensayos in situ como veleta de corte o CPT o ensayos de laboratorio para obtener $s_{u}$. El resumen de los parámetros del suelo necesarios según el tipo de suelo se muestra en la Tabla 14.

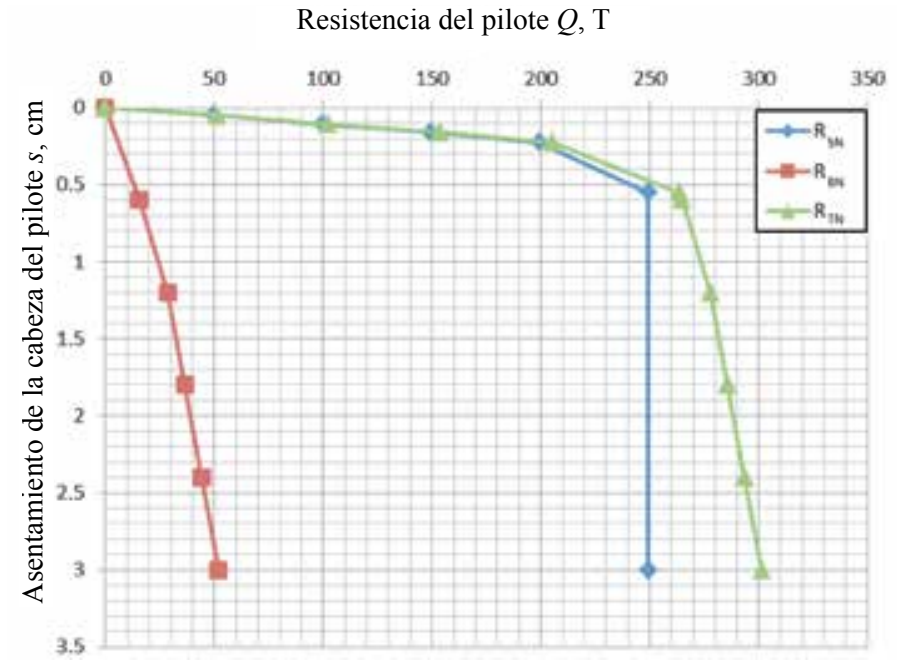

Figura 7: Curva resistencia versus asentamiento de acuerdo a FHWA 1999

Tabla 14: Parámetros de resistencia del suelo

\begin{tabular}{|c|c|c|c|c|}
\hline Estrato & suelo & $N_{60}$ & $s_{u^{\prime}} \mathrm{kPa}$ & $q_{c}, \mathrm{MPa}$ \\
\hline 1 & cohesivo & 3 & 25 & - \\
\hline 2 & cohesivo & 7 & 40 & - \\
\hline 3 & cohesivo & 23 & 153 & - \\
\hline 4 & no cohesivo & 32 & - & 9.6 \\
\hline 5 & no cohesivo & 32 & - & 12.8 \\
\hline
\end{tabular}

Una vez que se tienen los valores de resistencia de los ensayos, se utilizan directamente las Tablas 2 a 6 para obtener las resistencias últimas por punta y fuste. Las Tablas 15 a 18 resumen los cálculos y resultados para obtener la resistencia por fuste en estratos cohesivos y no cohesivos.

Tabla 15: Resistencia por fuste en suelo cohesivo

\begin{tabular}{|c|c|c|c|c|c|}
\hline Estrato & $A_{m \imath^{\prime}} \mathrm{m}^{2}$ & $S_{u^{\prime}} \mathrm{MPa}$ & $\tau_{m i^{\prime}} \mathrm{MPa}$ & $Q_{R S}, \mathrm{MN}$ & $Q_{R S} \mathrm{~T}$ \\
\hline 1 & 2.82 & 25 & 0.025 & 0.070 & 7.06 \\
\hline 2 & 5.65 & 40 & 0.028 & 0.158 & 15.8 \\
\hline 3 & 5.08 & 153 & 0.051 & 0.259 & 25.9 \\
\hline
\end{tabular}

Tabla 16: Resistencia por fuste en suelo no cohesivo

\begin{tabular}{|c|c|c|c|c|c|}
\hline Estrato & $A_{m i}, \mathrm{~m}^{2}$ & $q_{\mathcal{c}}, \mathrm{MPa}$ & $\tau_{m,}, \mathrm{MPa}$ & $Q_{R S} \mathrm{MN}$ & $Q_{R S} \mathrm{~T}$ \\
\hline 4 & 13.00 & 9.6 & 0.077 & 1.001 & 100.1 \\
\hline 5 & 11.12 & 12.8 & 0.102 & 1.134 & 113.4 \\
\hline
\end{tabular}


La resistencia por fuste total es de $Q_{R S}=262.4 \mathrm{~T}$. Para estimar la resistencia por punta del estrato soportante no cohesivo se utiliza la Tabla 2.

Tabla 17: Resistencia por punta en suelo no cohesivo

\begin{tabular}{|c|c|c|c|c|}
\hline$s / D$ & $s, \mathrm{~cm}$ & $\sigma_{S}, \mathrm{MPa}$ & $Q_{S S} \mathrm{MN}$ & $Q_{S S} \mathrm{~T}$ \\
\hline 0.02 & 1.2 & 0.896 & 0.253 & 25.3 \\
\hline 0.03 & 1.8 & 1.152 & 0.325 & 32.5 \\
\hline 0.1 & 6.0 & 2.56 & 0.723 & 72.3 \\
\hline
\end{tabular}

La capacidad axial del pilote $Q_{\mathrm{T}}$ se calcula como combinación de la resistencia por fuste y punta $Q_{\mathrm{T}}=334.8 \mathrm{~T}$.

Tabla 18: Capacidad axial total según DIN 4014

\begin{tabular}{|c|c|c|}
\hline$Q_{R S}, \mathrm{~T}$ & $Q_{S S}, \mathrm{~T}$ & $Q_{p}, \mathrm{~T}$ \\
\hline 262.4 & 72.3 & 334.8 \\
\hline
\end{tabular}

El asentamiento vertical asociado a los estados límites y las resistencias calculadas se obtienen de las expresiones (6) a (8). El asentamiento vertical movilizado por el fuste $s_{r g}$, está en función de la resistencia última por fricción del fuste y corresponde a $s_{r g}=1.81 \mathrm{~cm}$. El asentamiento vertical movilizado por la punta se muestra en la Tabla 17 y está dado a partir de los estados límite considerados en la DIN 4014. La capacidad última de resistencia en la base es movilizada por un asentamiento de la cabeza del pilote de un $10 \%$ de diámetro del pilote. La Figura 8 muestra la curva de resistencia versus asentamiento del pilote basada en los cálculos realizados previamente.

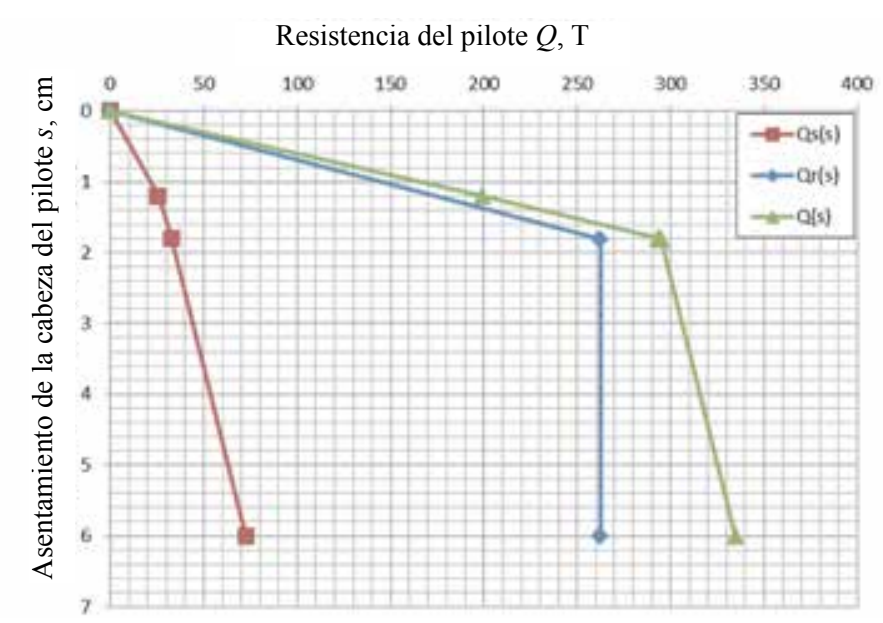

Figura 8: Curva resistencia versus asentamiento según DIN 4014

\section{Comparación de resultados}

Al comparar los resultados de la capacidad axial última estimada del pilote por ambas normas se obtiene el gráfico mostrado en la Figura 9.

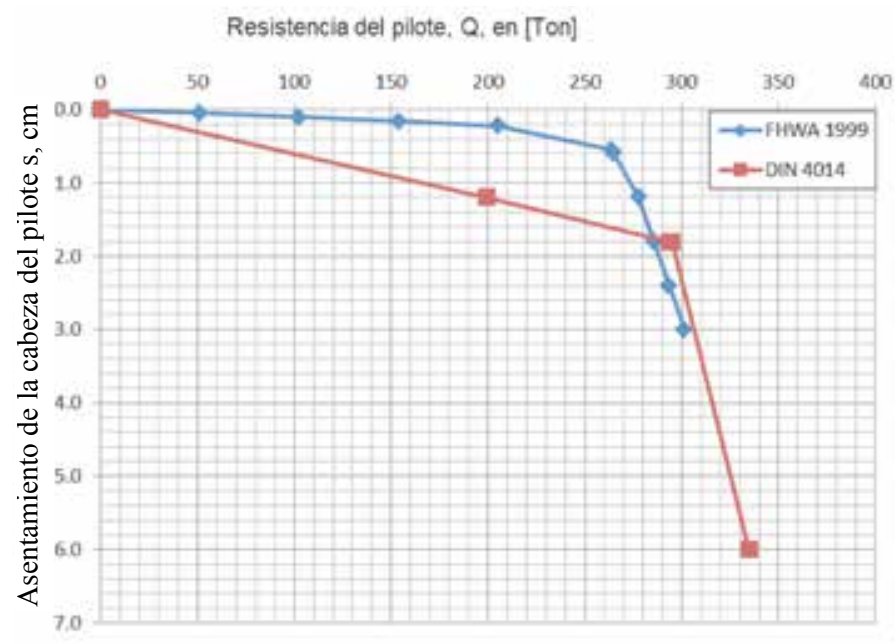

Figura 9: Comparación de resultados obtenidos por FHWA 1999 y DIN 4014

La diferencia relativa de resistencia última entre ambas normativas considerando un asentamiento de $3 \mathrm{~cm}$ en la cabeza del pilote como estado último de deformación (FHWA 1999) es cercano al 1\%. Esta diferencia es considerablemente pequeña para el rango de incertidumbre con el que se trabaja. No siempre se dispone de todos los valores de los parámetros necesarios para aplicar directamente los métodos. Es por ello que se debe tener precaución al utilizar tablas y fórmulas que correlacionen parámetros de suelo. El pilote estudiado es considerado un pilote de fuste, lo cual quiere decir que la mayoría de la resistencia es proporcionada por la fricción que se genera entre subsuelo y pilote.

Por otro lado existe una clara diferencia en la variación de la resistencia con el asentamiento, llegando a ser de 6 veces para $200 \mathrm{~T}$.

\section{Conclusiones}

La utilización del sistema de pilotes CFA o de hélice continua es una alternativa que presenta grandes beneficios, sobre todo por su rapidez y economía constructiva. Se debe tener en cuenta que para lograr los altos rendimientos 
que este sistema de fundación es capaz de proporcionar, es fundamental una adecuada exploración geotécnica incorporando ensayos CPT, veleta de corte o de laboratorio para determinar directamente $q_{c}$ y $s_{u}$ y no indirectamente del SPT. Según las primeras experiencias en Chile con este método, se logran rendimientos de entre 100 a $250 \mathrm{ml} /$ día.

Los requisitos de los materiales como acero y hormigón no difieren mucho de los requeridos por el sistema de pozos perforados convencionales, ampliamente utilizados en Chile. Sin embargo, a diferencia de éstos, es necesario que al comenzar con la perforación todo el hormigón requerido se encuentre en obra y listo para ser colocado. La perforación dura escasos minutos y al comenzar el hormigonado, éste debe terminarse en un solo proceso continuo.

La norma DIN 4014 trata el diseño de pilotes CFA dentro del diseño de pilotes pre-excavados, en el cual se determina la resistencia del pilote mediante tablas de origen empírico predeterminadas, que reflejan las condiciones de un suelo favorable. Esta norma puede ser utilizada cuando no se disponga de pruebas de carga ni experiencia en el lugar. La FHWA 1999, al igual que la DIN 4014, considera el diseño de los pilotes CFA dentro del diseño de los pilotes pre-excavados, lo cual tiende a subestimar la capacidad de un pilote CFA frente a un pilote perforado. De esta manera la aplicación de esta norma permite mayores grados de seguridad.

\section{Agradecimientos}

Los autores agradecen a Pilotes Terratest por facilitar los antecedentes relativos a los pilotes CFA de la obra en Coronel que permitieron el estudio del caso práctico en esta investigación.

\section{Referencias}

Bauer (2011). BG 50: Rotary Drilling Ring. Retrieved from: http://www.bauer.de/en/bma/products/drilling_rigs/premium _ line/bg50_bt180.html
Brown, D.A., Dapp, S.D., Thompson, W.R. and Lazarte, C.A. (2007). Design and Construction of Continuous Flight Auger (CFA) Piles. Geotechnical Engineering Circular No. 8. FHWA, Washington DC

Candogan, A. (2009). The Art and Practice of Foundation Engineering. Ali Candogan, Estambul.

Coduto, D.P., Yeung M.R. and Kitch, W.A. (2011). Geotechnical Engineering: Principles and Practices. Pearson Education, New York

DIN (1990). DIN 4014: Bored cast-in-place piles: formation, design and bearing capacity. Germany

INN (2006). NCh 204 Of. 2006: Acero- Barras laminadas en caliente para hormigón armado. Instituto Nacional de Normalización, Santiago

Kempfert, H., Dieter, K. and Smoltczyk, U. (2003). Pile foundations. In U. Smoltczyk, Geotechnical Engineering Handbook Volume 3: Elements and Structures (pp. 83-227). Ernst \& Sohn, Berlin

O’Neill, M.W. and Reese, L.C. (1999). Drilled Shafts: Construction Procedures and Design Methods. Report No. FHWA-IF-99-025. Federal Highway Administration, Washington DC

Pilotes Terratest (2009). Pilotes de Hélice Continua - CFA. Retrieved from: http://www.terratest.cl/documentos/CAT-CFA01-rev\%202.pdf

Reese, L.C. and O'Neill, M.W. (1989). New design method for drilled shafts from common soil and rock tests. Proceedings of Foundation Engineering: current principles and practices. ASCE Vol. 2, 1026-1039

Stocker, M. and Waltz, B. (2003). Bored pile walls, diaphragm walls, cut-off walls. In U. Smoltczyk, Geotechnical Engineering Handbook Volume 3: Elements and Structures (pp. 409-450). Ernst \& Sohn, Berlin

Terzaghi, K., Peck, R. B. and Mesri, G. (1996). Soil Mechanics in Engineering Practice. John Wiley \& Sons, New York.

Tomlinson, M. and Woodward, J. (2007). Pile Design and Construction Practice. Taylor \& Francis, New York 